\title{
Radion Induced Spontaneous Baryogenesis
}

\author{
G.L. Alberghi* R. Casadio ${ }^{\dagger}$ and A. Tronconi ${ }^{\ddagger}$ \\ Dipartimento di Fisica, Università di Bologna, and \\ Istituto Nazionale di Fisica Nucleare, Sezione di Bologna, Italy
}

October 31, 2018

\begin{abstract}
We describe a possible scenario for spontaneous baryogenesis given by the Randall-Sundrum brane-world model with two branes and a radion stabilizing potential. It is known that the cosmological expansion of the Universe can be recovered in this brane-world model by adding matter on the branes, and time-reversal is then broken. We show here that, as a consequence of such a time dependence of the system, the effective coupling of the radion with matter fields generates a chemical potential with opposite signs for baryons and anti-baryons and spontaneous baryogenesis is therefore induced naturally and self-consistently.
\end{abstract}

\section{Introduction}

One of the most peculiar features of our Universe is the observed baryonic asymmetry. This can be conveniently characterized by the dimensionless number

$$
\frac{n_{B}}{s} \equiv \eta \simeq 10^{-10}
$$

where $n_{B} \equiv n_{b}-n_{\bar{b}}$ is the difference between the baryon and anti-baryon densities and $s$ is the density of entropy. The consistency of primordial nucleosynthesis, which yields some of the most precise results in the standard model of cosmology, requires that $\eta$ took the above value at the time when the light elements (i.e., ${ }^{3} \mathrm{He},{ }^{4} \mathrm{He}$, and ${ }^{7} \mathrm{Li}$ ) were produced, and it is believed to have then remained the same up to the present epoch.

The necessary conditions for generating the baryonic asymmetry in quantum field theory were formulated by Sacharov in 1967 [1] (see also Ref. [2]) and can be summarized as follows:

1. Different interactions for particles and antiparticles, or, in other words, a violation of the $\mathrm{C}$ and $\mathrm{CP}$ symmetries;

2. Non-conservation of the baryonic charge;

3. Departure from thermal equilibrium.

\footnotetext{
*e-mail: alberghi@bo.infn.it

†e-mail: casadio@bo.infn.it

${ }^{\ddagger}$ e-mail: tronconi@bo.infn.it
} 
The last condition results from an application of the CPT theorem $[3,4]$. In fact, CPT invariance of quantum field theory in a static Minkowski space-time ensures that the energy spectra for baryons and anti-baryons are identical, leading consequently to identical distributions at thermal equilibrium. This explains why the baryon number asymmetry was required to be generated out of thermal equilibrium.

The so called mechanism of spontaneous baryogenesis $[5,6]$ uses the natural (strong) CPT non-invariance of the Universe during its early history to bypass this third condition. We know that an expanding Universe at finite temperature violates both Lorentz invariance and time reversal, and this can lead to effective CPT violating interactions [3, 4]. Thus the cosmological expansion of the early Universe leads us naturally to examine the possibility of generating the baryon asymmetry in thermal equilibrium. The main ingredient for implementing this mechanism is a scalar field $\phi$ with a derivative coupling to the baryonic current. If the current is not conserved and the time derivative of the scalar field has a non-vanishing expectation value, an effective chemical potential with opposite signs for baryons and anti-baryons is generated leading to an asymmetry even in thermal equilibrium.

The brane-world model with two branes proposed by Randall and Sundrum (RS) in Ref. [7] contains a metric degree of freedom called the radion which determines the distance between the two branes and appears as a scalar field $\phi$ on the branes. Cosmological solutions have also been examined rather extensively in this context. In particular, it has been shown that, when matter is added on one (or both) of the two branes, the standard Friedmann equation for the scale factor of the Universe is recovered (with possible corrections) provided the radion is suitably stabilized (see for example Refs. [8]-[11] and References therein). In this brane-world model, we therefore have both a scalar field (the radion) and the cosmological evolution as required by spontaneous baryogenesis, and we shall show that the radion field does in fact couple differently with baryons and anti-baryons. This scenario might therefore naturally reproduce the observed baryonic asymmetry ${ }^{1}$.

In Section 2, we review in some details the mechanism of spontaneous baryogenesis. The cosmological solutions in the RS framework are discussed in Section 3 where the process of spontaneous baryogenesis driven by the radion is presented in general. Some more specific examples are also reported in Section 4. We then conclude and comment on our results.

We shall use units with $c=\hbar=k_{\mathrm{B}}=1$, where $k_{\mathrm{B}}$ is the Boltzmann constant.

\section{Spontaneous Baryogenesis}

To illustrate the mechanism of spontaneous baryogenesis (see, e.g., Refs. [5, 6] and [19]-[21]) let us consider a theory in which a neutral scalar field $\phi$ is coupled to the baryonic current $J_{B}^{\mu}$ according to the Lagrangian density

$$
L_{\mathrm{int}}=\frac{\lambda^{\prime}}{M_{\mathrm{c}}} J_{B}^{\mu} \partial_{\mu} \phi
$$

where $\lambda^{\prime}$ is a coupling constant and $M_{c}$ is a cut-off mass scale in the theory (presumably smaller than the Planck mass $M_{\mathrm{Pl}}$ ). Let us assume that $\phi$ is homogeneous, so that only the time

\footnotetext{
${ }^{1}$ Other mechanisms for baryogenesis in the context of brane-world models have been recently analyzed, e.g. in Refs. [12]-[18].
} 
derivative term contributes,

$$
L_{\text {int }}=\frac{\lambda^{\prime}}{M_{\mathrm{c}}} \dot{\phi} n_{B} \equiv \mu(t) n_{B}
$$

where $n_{B}=J_{B}^{0}$ is the baryon number density and $\mu(t)$ is to be regarded as an effective timedependent chemical potential. This interpretation (see Ref. [22]) is valid if the current $J_{B}^{\mu}$ is not conserved (otherwise one could integrate the interaction term away) and if $\phi$ behaves as an external field which develops a slowly varying time derivative $\langle\dot{\phi}\rangle \neq 0$ as the Universe expands. Since the chemical potential $\mu$ enters with opposite signs for baryons and anti-baryons, we have a net baryonic charge density in thermal equilibrium at the temperature $T$,

$$
n_{B}(T ; \xi)=\int \frac{\mathrm{d}^{3} k}{(2 \pi)^{3}}[f(k, \mu)-f(k,-\mu)]
$$

where $\xi \equiv \mu / T$ is regarded as a parameter, and

$$
f(k, \mu)=\frac{1}{\exp \left[\left(\sqrt{k^{2}+m^{2}}-\mu\right) / T\right] \pm 1}
$$

is the phase-space thermal distribution ${ }^{2}$ for particles with rest mass $m$ and momentum $k$. For $|\xi| \ll 1$ we may expand Eq. (2.4) in powers of $\xi$ to obtain

$$
n_{B}(T ; \mu)=\frac{g T^{3}}{6} \xi+O\left(\xi^{2}\right)
$$

where $g$ is the number of degrees of freedom of the field corresponding to $n_{B}$. Upon substituting in for the expression of $\mu$, one therefore finds

$$
n_{B}(T ; \mu) \simeq \frac{\lambda^{\prime} g}{6 M_{\mathrm{c}}} T^{2}\langle\dot{\phi}\rangle
$$

Regardless of the specific mechanisms which break baryon number conservation, we assume that there is a temperature $T_{\mathrm{F}}$ at which the baryon number violating processes become sufficiently rare so that $n_{B}$ freezes out ( $T_{\mathrm{F}}$ will in fact be called the freezing temperature). Once this temperature is reached as the universe cools down, one is left with a baryonic asymmetry whose value is given by Eq. (2.6) evaluated at $T=T_{\mathrm{F}}$. The value of the parameter $\eta$ remains unchanged in the subsequent evolution.

\section{Radion Induced Spontaneous Baryogenesis}

We have discussed how the mechanism of spontaneous baryogenesis may explain the observed baryonic asymmetry. We shall now argue that it might occur naturally in brane-world models. In particular, we shall consider the five-dimensional RS model of Ref. [7] perturbed by matter on one or both branes [9]-[11]. The reader is referred to Ref. [8] for more details on the framework and notation used hereafter.

\footnotetext{
${ }^{2}$ Of course, the plus sign is for fermions and the minus sign for bosons.
} 
In this model the metric can be written in the form

$$
\begin{aligned}
\mathrm{d} s^{2} & =n^{2}(y, t) \mathrm{d} t^{2}-a^{2}(y, t)\left[\left(\mathrm{d} x^{1}\right)^{2}+\left(\mathrm{d} x^{2}\right)^{2}+\left(\mathrm{d} x^{3}\right)^{2}\right]-b^{2}(y, t) \mathrm{d} y^{2} \\
& \equiv \tilde{g}_{A B}(x, y) \mathrm{d} x^{A} \mathrm{~d} x^{B}
\end{aligned}
$$

where $t$ is the time, $x^{i}$ are the spatial coordinates along the branes and $y$ is the extra-dimensional coordinate. In this formalism, the Planck brane is conventionally located at $y=0$ and the $\mathrm{TeV}$ brane at $y=1 / 2$. The Einstein equations are given by $G_{A B}=\kappa^{2} T_{A B}$, where $\kappa^{2}=1 /\left(2 M^{3}\right)$ and $M$ is the five-dimensional Planck mass. The energy-momentum tensor $T_{A B}$ contains a contribution from the bulk cosmological constant $\Lambda$ of the form $T_{A B}^{\text {bulk }}=\Lambda \tilde{g}_{A B}$ and a contribution from the matter on the two branes,

$$
\begin{aligned}
T_{A}^{B} \text { branes }= & \frac{1}{b} \delta(y) \operatorname{diag}\left[V_{*}+\rho_{*}, V_{*}-p_{*}, V_{*}-p_{*}, V_{*}-p_{*}, 0\right] \\
& +\frac{1}{b} \delta(y-1 / 2) \operatorname{diag}[V+\rho, V-p, V-p, V-p, 0],
\end{aligned}
$$

where $V_{*}$ is the (positive) tension of the Planck brane and $V$ the (negative) tension on the $\mathrm{TeV}$ brane. We have correspondingly denoted by $\rho_{*}$ and $p_{*}$ the density and pressure of the matter localized on the positive tension (Planck) brane (assuming an equation of state of the form $\left.p_{*}=w_{*} \rho_{*}\right)$ and by $\rho$ and $p$ the density and pressure of the matter on the negative tension ( $\left.\mathrm{TeV}\right)$ brane. Once a stabilizing potential for the radion is included, the stress-energy tensor picks up an additional term and the solution of the Einstein equations may be written as a perturbation of the usual RS solution,

$$
n(y)=a(y)=e^{-m_{0} b_{0}|y|},
$$

with $V_{*}=6 m_{0} / \kappa^{2}=-V$ and $\Lambda=-6 m_{0}^{2} / \kappa$. We also recall that the constants $b_{0}$ and $m_{0}$ determine the effective four-dimensional Planck mass as $\left(8 \pi G_{N}\right)^{-1}=M_{\mathrm{Pl}}^{2}=\left(1-\Omega_{0}^{2}\right) / \kappa^{2} m_{0}$, where $\Omega_{0} \equiv e^{-m_{0} b_{0} / 2}$.

In order to obtain an effective action for the four-dimensional theory, one perturbs the metric about the RS solution in the form

$$
\begin{aligned}
& a(t, y)=a(t) e^{-m_{0} b(t)|y|}[1+\delta a(y, t)] \\
& n(t, y)=e^{-m_{0} b(t)|y|}[1+\delta n(y, t)] \\
& b(t, y)=b(t)[1+\delta b(y, t)]
\end{aligned}
$$

and drops the metric perturbations which contribute only to second order in $\delta a, \delta n$ and $\delta b$ (see Refs. [9]-[11] for the effects of the latter). It is then useful to introduce the notation $\Omega(y, b(t))=e^{-m_{0} b(t)|y|}$ and $\Omega_{b} \equiv \Omega(1 / 2, b(t))\left(\Omega_{b}\right.$ evaluated at $b=b_{0}$ is then given by $\left.\Omega_{0}\right)$. By integrating over the fifth dimension, one obtains an effective action for the radion field. Further, upon examining the equations of motion for $b(t)$, one notes that, since $\Omega_{b}$ depends on $b$, the presence of matter on the two branes generates an effective potential for $b(t)$ given by

$$
V_{\mathrm{eff}}(b)=V_{r}(b)+\frac{f^{4}(b)}{4}\left[\rho_{*}-3 p_{*}+(\rho-3 p) \Omega_{b}^{4}\right],
$$


with

$$
f(b)=\left(\frac{1-\Omega_{0}^{2}}{1-\Omega_{b}^{2}}\right)^{1 / 2} .
$$

The function $V_{r}=V_{r}(b(t))$ is the potential which would stabilize the radion at the value $b=b_{0}$ in the absence of matter. It can therefore be expanded near its minimum as ${ }^{3}$

$$
V_{r}(b)=\frac{1}{4} m_{r}^{2}\left(\frac{m_{0} b_{0}}{1-\Omega_{0}^{2}}\right)^{2} \Omega_{0}^{2} M_{\mathrm{Pl}}^{2}\left(\frac{b-b_{0}}{b_{0}}\right)^{2},
$$

where

$$
m_{r}^{2}=\frac{2\left(f^{4} V_{r}\right)^{\prime \prime}\left(b_{0}\right)}{3 m_{0}^{2} M_{\mathrm{Pl}}^{2} \Omega_{0}^{2}}\left(1-\Omega_{0}^{2}\right)^{2},
$$

and $m_{r}$ is the effective radion mass. Thus the radion, in the presence of matter on the two branes, is stabilized to a shifted value $b_{0}+\Delta b$ determined by

$$
\frac{\Delta b}{b_{0}}=\frac{1}{3}\left(\frac{1-\Omega_{0}^{2}}{m_{0} b_{0}}\right) \frac{\rho-3 p+\Omega_{0}^{2}\left(\rho_{*}-3 p_{*}\right)}{m_{r}^{2} \Omega_{0}^{2} M_{\mathrm{Pl}}^{2}},
$$

where $\Delta b$ is the distance between the minima of $V_{\text {eff }}$ with and without matter.

From the above expression, we see that, since the trace of the stress-energy tensor vanishes for radiation, even if the Universe on the $\mathrm{TeV}$ brane is in a radiation dominated era $(\rho \simeq 3 p)$, the radion evolution is determined by the behavior of massive matter on the $\mathrm{TeV}$ and Planck branes. This is the essential ingredient which allows for the possibility of inducing spontaneous baryogenesis. We note that the factor $\Omega_{0}^{2}$ in front of $\rho_{*}$ would make this term negligible for comparable energy densities on the $\mathrm{TeV}$ and Planck branes, but the fact that the natural energy scale on the Planck brane is of the order of $M_{\mathrm{Pl}}$ may nonetheless allow for a relevant contribution to baryogenesis from the Planck brane.

Let us now assume that the high energy Lagrangian for matter on the TeV brane contains an interaction term of the form given in Eq. (2.2),

$$
L_{\mathrm{int}}=\lambda^{\prime} m_{0} \dot{b} n_{B}
$$

where $\dot{b}$ now plays the role of $\langle\dot{\phi}\rangle$. Such a term is the same as that in Eq. (4.30) of Ref. [8]. On using Eq. (3.9) to estimate $\dot{b} \simeq \dot{\Delta b}$, we finally obtain

$$
\eta=\frac{m_{0} \dot{b}}{T}=\frac{1}{m_{r}^{2} \Omega_{0}^{2} M_{\mathrm{Pl}}^{2}}\left(\frac{1}{T}\right) \frac{\mathrm{d}}{\mathrm{d} t}\left[(\rho-3 p)+\Omega_{0}^{2}\left(\rho_{*}-3 p_{*}\right)\right] .
$$

Due to the expansion of the Universe, the time derivative on the right hand side of this equation will in general acquire a non vanishing (expectation) value and the baryonic symmetry is therefore dynamically broken in the model. We also note that the radion field is likely very massive ${ }^{4}$, and one can then assume that the radion follows instantaneously any changes of the matter density.

\footnotetext{
${ }^{3}$ This expression follows from Eq. (4.12) of Ref. [8] by defining $\sqrt{3 / 2} \phi / \Lambda_{W}=m_{0} b$ (where $\Lambda_{W}=\Omega_{0} M_{\mathrm{Pl}} \simeq$ $1 \mathrm{TeV})$.

${ }^{4}$ For example, if the radion is stabilized by the Goldberger-Wise mechanism [23], one has $m_{r} \simeq 1 \mathrm{TeV}$.
} 


\section{Examples}

In order to complete our analysis, we shall now estimate the baryonic asymmetry (3.11) at the freezing temperature $T_{\mathrm{F}}$ in three specific scenarios:

1. If the effect of matter on the Planck brane is negligible in Eq. (3.11), the condition to generate the observed baryonic asymmetry (1.1) can be estimated as

$$
\left.\frac{1}{T} \frac{\mathrm{d}}{\mathrm{d} t}(\rho-3 p)\right|_{T_{\mathrm{F}}} \gtrsim 10^{-10} \mathrm{TeV}^{4} .
$$

Let $\rho_{m}$ be the energy density of any non-traceless component of the energy momentum tensor. By using the continuity equation and the Friedmann equation for a radiation dominated Universe up to an energy density of the order of $1 \mathrm{TeV}^{4}$, which is roughly the limit of validity for the RS model, one obtains the requirement

$$
\rho_{m} \gtrsim 10^{-6} \mathrm{TeV}^{4}
$$

for this mechanism to produce sufficient baryonic asymmetry.

2. Since the Planck brane remains hidden, one can allow for a very large term proportional to $\dot{\rho}_{*}$ in Eq. (3.11) (we recall that matter energy on the Planck brane is allowed up to the Planck scale). The radion velocity would hence be larger than in the previous case, and the freezing temperature correspondingly lower. The bound in this case is

$$
\left.\frac{1}{T} \frac{\mathrm{d}}{\mathrm{d} t}\left(\rho_{*}-3 p_{*}\right)\right|_{T_{\mathrm{F}}} \gtrsim 10^{-42} M_{\mathrm{Pl}}^{4} .
$$

3. Another possibility is to consider the stage when the radion is still stabilizing towards the equilibrium value $b_{0}$ and the effect of matter on the branes is negligible. A typical radion velocity would be larger than in both previous cases, $m_{0} \dot{b} \simeq H(T) M_{\mathrm{Pl}} / \Lambda_{W}$, and

$$
T \gtrsim 10^{2} \mathrm{eV}
$$

which allows the widest range of temperature among the three possibilities outlined.

Of course, the above list is not exhaustive and one could consider many other situations. For example, one could include more bulk fields or different couplings between the radion and brane fields. A complete analysis of all possible cases however goes beyond the scope of the present work and will not be given here.

\section{Conclusions}

We have shown that the perturbations induced by the addition of matter on one (or both) of the two branes of a cosmological RS model with a stabilizing potential for the radion naturally lead to a non-vanishing expectation value for the velocity of the radion field. Since the latter couples with the baryonic current on the branes, this naturally induces the onset of spontaneous baryongenesis, as described by the general formula (3.11).

Having outlined the main ideas in the present paper, the next step would be to analyze all possible scenarios. For specific cases, it may in fact be possible to reproduce the observed baryonic asymmetry $\eta$ in Eq. (1.1). Conversely, the required value of $\eta$ can be viewed as a constraint that brane-world models must satisfy. 


\section{Acknowledgments}

R.C. would like to thank G. Lambiase for comments.

\section{References}

[1] A.D. Sakharov, Pis'ma Z. Eksp. Teor. Fiz. 5 (1967) 32 [JETP Lett. 5 (1967) 24].

[2] S. Dimopoulos and L. Susskind, Phys. Rev. D 18 (1978) 4500; Phys. Lett. B 81 (1979) 416.

[3] R.F. Streater and A.S. Wightman, PCT, spin and statistics, and all that, Redwood City, USA, Addison-Wesley (1989).

[4] R. Jost, The general theory of quantized fields, Providence, American mathematical society, 1965 .

[5] A.G. Cohen and D.B. Kaplan, Phys. Lett. B 199 (1987) 251.

[6] A.G. Cohen and D.B. Kaplan, Nucl. Phys. B 308 (1988) 913.

[7] L. Randall and R. Sundrum, Phys. Rev. Lett. 83 (1999) 3370.

[8] C. Csaki, M. Graesser, L. Randall and J. Terning, Phys. Rev. D 62 (2000) 045015;

[9] G. L. Alberghi, D. Bombardelli, R. Casadio and A. Tronconi, Phys. Rev. D 72 (2005) 025005 .

[10] G. L. Alberghi and A. Tronconi, Phys. Rev. D 73 (2006) 027702.

[11] J.M. Cline and J. Vinet, JHEP 02 (2002) 042.

[12] M.R. Martin, Phys. Rev. D 67 (2003) 083503.

[13] T. Matsuda, Phys. Rev. D 65 (2002) 103501.

[14] M. Bastero-Gil, E.J. Copeland, J. Gray, A. Lukas and M. Plumacher, Phys. Rev. D 66 (2002) 066005.

[15] D.J.H. Chung and T. Dent, Phys. Rev. D 66 (2002) 023501.

[16] A. Mazumdar and A. Perez-Lorenzana, Phys. Rev. D 65 (2002) 107301.

[17] R. Allahverdi, K. Enqvist, A. Mazumdar and A. Perez-Lorenzana, Nucl. Phys. B 618 (2001) 277.

[18] A. Masiero, M. Peloso, L. Sorbo and R. Tabbash, Phys. Rev. D 62 (2000) 063515.

[19] A. De Felice, S. Nasri and M. Trodden, Phys. Rev. D 67 (2003) 043509.

[20] M. Li, X. Wang, B. Feng, and X. Zhang, Phys. Rev. D 65 (2002) 103511.

[21] M. Li and X. Zhang, Phys. Lett. B 573 (2003) 20. 
[22] A.D. Dolgov, Phys. Rept. 222 (1992) 309.

[23] W.D. Goldberger and M.B. Wise, Phys. Rev. D 60 (1999) 107505.

[24] N. Bralic, D. Cabra and F.A. Schaposnik, Phys. Rev. D 50 (1994) 5314. 